\title{
Variabilidad fenotípica de la fibrosis quística: reporte de 2 gemelos con la mutación F508/F508
}

\author{
MARÍA FERNANDA HERNÁNDEZ-AMARIS ${ }^{1}$, \\ ANA MARÍA GÓMEZ-VÁSQUEZ ${ }^{2}$, HARRY PACHAJOA H. ${ }^{1,2}$ \\ 1. Centro de Investigaciones en Anomalías Congénitas y Enfermedades Raras (CIACER), Universidad Icesi. Cali, Colombia. \\ 2. Fundación Clínica Valle del Lili. Cali, Colombia.
}

\begin{abstract}
Phenotypic variability of cystic fibrosis: case report of twins with F508/F508 mutation

Introduction: Cystic fibrosis (CF) is an autosomal recessive disease caused by a mutation in the CFTR gene, resulting in an alteration of a protein involved in sodium and chloride transport in the apical plasma membrane of epithelial cells in respiratory and intestinal tracts. It primarily presents respiratory compromise, affecting other systems in different ways. Meconium ileus is a gastrointestinal manifestation that occurs in $10-20 \%$ of patients, which is not entirely attributable to a specific CFTR mutation. Objective: To report a case of monozygotic twins diagnosed with CF (F508) in whom phenotypic variation is evident based on the expression of meconium ileus, showing that there are external modifiers in the development of this complication. Case report: monoamniotic monochorionic twin pregnancy which resulted in preterm births. One of the patient presented meconium ileus at birth leading to CF suspicion and establishing the diagnosis by (F508/F508) molecular analysis in both twins. Conclusion: Phenotypic variability in these twins supports the hypothesis proposed by different authors that there are other gene expression-modulation factors of the disease as well as environmental modifiers that must be taken into account when dealing with this disease.
\end{abstract}

(Key words: Cystic fibrosis, ileus, meconium, twin pregnancy).

Rev Chil Pediatr 2014; 85 (4): 470-475

\section{RESUMEN}

Introducción: La fibrosis quística $(\mathrm{FQ})$ es una enfermedad autosómica recesiva debida a una mutación en el gen CFTR, resultando en una alteración de una proteína implicada en el transporte de sodio y cloro en la membrana apical de células del epitelio respiratorio y gastrointestinal; presenta principalmente compromiso respiratorio, afectando otros sistemas de manera variable. El íleo meconial es una manifestación gastrointestinal presente en $10-20 \%$ de los pacientes, no del todo atribuible una mutación específica del CFTR. Objeti-

Recibido el 08 de octubre de 2013, última versión aceptada para publicación el 27 de mayo de 2014.

Potenciales conflictos de interés: Este trabajo cumple con los requisitos sobre consentimiento /asentimiento informado, comité de ética, financiamiento, estudios animales y sobre la ausencia de conflictos de intereses según corresponda.

Correspondencia a:

Harry Pachajoa $\mathrm{H}$.

E-mail. hmpachajoa@icesi.edu.co 
vo: Reportar un caso de dos gemelos monocigóticos con diagnóstico de FQ ( $\Delta \mathrm{F} 508)$, en quienes es evidente la variabilidad fenotípica en cuanto a la expresión de íleo meconial, poniendo de manifiesto que existen modificadores externos a la mutación en el desarrollo de esta complicación. Reporte de caso: Gemelos producto de embarazo monocorial monoamniotico, nacidos pretérmino, uno de ellos presenta íleo meconial al nacimiento lo que lleva al estudio de $\mathrm{FQ}$, estableciendo el diagnóstico por análisis molecular $(\Delta \mathrm{F} 508 / \Delta \mathrm{F} 508)$ en ambos gemelos. Conclusión: La variabilidad fenotípica en estos gemelos apoya la hipótesis planteada por diferentes autores de que existen otras factores genéticos moduladores de la expresión de la enfermedad, así como modificadores ambientales, que deben ser tenidos en cuenta a la hora de abordar esta enfermedad.

(Palabras clave: Fibrosis quística, íleo, meconial, embarazo gemelar).

Rev Chil Pediatr 2014; 85 (4): 470-475

\section{Introducción}

La fibrosis quística (FQ) es una enfermedad autosómica recesiva que afecta niños y adultos, caracterizada principalmente por compromiso pulmonar, aunque puede afectar también páncreas, hígado y tracto gastrointestinal $^{1}$; tiene una prevalencia de 1 en 2.000 a 2.500 nacidos vivos en poblaciones caucásicas, representando la enfermedad autosómica recesiva más común en esta población. Es causada por una mutación del gen que codifica la proteína reguladora de la conductancia transmembrana de la fibrosis quística (CFTR, por sus siglas en inglés), manifestándose en una disfunción de la proteína reguladora, asociada a un transporte anormal de sodio y cloro en el epitelio, que como consecuencia se presenta con secreciones espesas y viscosas que predisponen a infecciones a nivel pulmonar ${ }^{2}$, además de repercusiones a nivel de los otros sistemas ya mencionados y las alteraciones del sudor, utilizadas como método diagnóstico de la enfermedad.

La mutación del CFTR más prevalente es la Delta-F508 ( $\triangle$ F508), dada por una deleción de fenilalanina en la posición 508. Esta mutación es responsable de aproximadamente el $70 \%$ de los pacientes con fibrosis quística en la población caucásica, llegando a estimarse que aproximadamente el $50 \%$ de todos los pacientes con fibrosis quística identificados como homocigotos para esta mutación tiene un ancestro en el norte de Europa ${ }^{3}$. Además este genotipo está generalmente asociado a las formas más graves de la enfermedad y al compromiso de páncreas y tracto gastrointestinal.
Dentro de las posibles manifestaciones gastrointestinales de la enfermedad se encuentra el íleo meconial, el cual se presenta como una de las manifestaciones iníciales de la enfermedad en 10 a $20 \%$ de los pacientes. A pesar de reconocerse el gen alterado causante de esta enfermedad, la variabilidad de presentación indica que existen factores reguladores o modificadores de la expresión de la enfermedad. El objetivo de este trabajo es reportar un caso de dos gemelos monocigóticos con diagnóstico de fibrosis quística confirmado por análisis molecular del gen CFTR, en quienes es evidente la variabilidad fenotípica en cuanto a la expresión de íleo meconial, poniendo de manifiesto que existen modificadores genéticos en el desarrollo de esta complicación.

\section{Caso clínico}

Se trata de dos hermanos gemelos recién nacidos pre término a las 35 semanas de edad gestacional productos de embarazo monocorial monoamniotico, madre niega consanguinidad parental y refiere antecedente de aborto espontáneo asociado a síndrome de Turner $(45, \mathrm{X} 0)$ confirmado por cariotipo fetal en su primer embarazo. Segundo embarazo normal, no se realizó cariotipo fetal, llevó a cabo adecuados controles prenatales y ecográficos sin evidencia de complicaciones hasta la semana 35; en este momento se identificó, mediante ecografía obstétrica, obstrucción y posible isquemia intestinal en el gemelo \#1. Dado lo anterior se programó para cesárea de emergencia.

Se obtuvo primer gemelo con peso de 2.520 
$\mathrm{g}$, talla de $47 \mathrm{~cm}$ con puntaje APGAR al primer minuto, 5 min y 10 min de 9-10-10 respectivamente, en quien se evidenció marcada distensión abdominal; y segundo gemelo con peso de $2.300 \mathrm{~g}$, talla de $47 \mathrm{~cm}$ y APGAR de 9-10-10.

Al nacimiento el gemelo \#2 se adaptó sin problemas, sin embargo, el gemelo \#1 presentó un episodio de apnea que se manejó mediante ventilación con presión positiva (VPP), a lo que respondió de manera satisfactoria. Se decidió paso de sonda nasogástrica, mediante la cual se drenó abundante líquido bilioso. Se trasladó a la unidad de cuidados intensivos neonatal y se adaptó a ventilación mecánica teniendo en cuenta el alto riesgo de broncoaspiración; entre los paraclínicos se realizó una radiografía de abdomen simple que mostró signos de obstrucción intestinal distal; y un enema de bario donde se evidenció microcolon. Se decidió manejo quirúrgico por laparotomía, con los siguientes hallazgos intraoperatorios: hipoplasia de vesícula biliar, hipoplasia intestinal, presencia de múltiples adherencias e íleo meconial que afectaba el íleon y el colon, con necrosis de $10 \mathrm{~cm}$ del Íleon proximal; posterior a liberación de adherencias y resección de segmento necrosado se cerró dejando yeyunostomía.

En los primeros 4 días postoperatorios recibió nutrición parenteral y posteriormente se inició nutrición enteral. Al persistir con motilidad intestinal disminuida y presentar alto gasto por estoma se decidió administración de agente proquinético y al sexto día postoperatorio se realizo un enema, posterior al cual presentó finalmente meconio.

Teniendo en cuenta la asociación de íleo meconial como una de las primeras manifestaciones de fibrosis quística, se decidió realizar estudio molecular en los dos hermanos. Se tomó muestra de ADN a partir de muestras de sangre, se realizó amplificación por reacción de polimerasa en cadena (PCR) con primers específicos ${ }^{4}$, se prepararon mezclas de reacción (amortiguador de PCR 1X, $\mathrm{MgCl} 2,1,5$ $\mathrm{mM}$, deoxinucleótidos trifosfato $0,04 \mathrm{mM}, 1 \mathrm{U}$ Taq ADN polimerasa, primers $0,5 \mathrm{pmol} / \mu \mathrm{l}$ y 100 ng de $\mathrm{ADN}$ ), encontrándose en ambos hermanos la mutación homocigótica $\Delta \mathrm{F} 508$.
El gemelo \#1 evolucionó satisfactoriamente, y tanto él cómo su hermano continuaron en manejo ambulatorio con controles de función pulmonar y manejo por gastropediatría, teniendo en cuenta la asociación de la mutación $\Delta \mathrm{F} 508 / \Delta \mathrm{F} 508$ con compromiso de vías biliares y tracto gastrointestinal.

\section{Discusión}

La Fibrosis Quística es una condición genética con patrón de herencia autosómico recesivo, asociada a una alta mortalidad con una prevalencia de 1 en 2.000 nacidos vivos entre la población caucásica, en quien se estima una de cada 25 personas es portadora asintomáti$\mathrm{ca}^{1,2,5}$. La proteína reguladora de conductancia transmembrana de la fibrosis quística es una proteína de 1.480 aminoácidos que forma canales de cloro regulados por cAMP. Esta proteína es codificada por el gen CFTR, un gen de 27 exones que se encuentra localizado en el cromosoma 7q31.3 $3^{3,6}$. Se han descrito más de 1.900 mutaciones a nivel de este gen, la más frecuente, responsable de cerca del $70 \%$ de los $\operatorname{casos}^{7}$, es conocida como F508 del $(\Delta \mathrm{F} 508)$, esta causa un defecto en el transporte de la proteína dificultando su llegada a la membrana apical $^{2,8,9}$. Siguiendo el patrón de la enfermedad, esta mutación es más prevalente en la población caucásica, estimándose que el $50 \%$ de todos los pacientes con FQ quienes son homocigotos para esta mutación tienen un ancestro del norte de Europa ${ }^{3}$.

La proteína CFTR es expresada en la membrana apical de las células que recubren el epitelio respiratorio, de los conductos pancreáticos, del sistema gastrointestinal, del tracto genitourinario y de las glándulas sudoríparas $^{2,3,10}$. Esta localización explica las manifestaciones clínicas de la enfermedad, tales como malabsorción de nutrientes secundaria a insuficiencia pancreática, falla pulmonar severa, hiponatremia, pólipos nasales, prolapso rectal, pancreatitis, colelitiasis y diabetes ${ }^{6,11-13}$. El diagnóstico Gold Standard basa principalmente en la medición de electrolitos en sudor mediante una prueba induciendo el sudor con pilocarpina, el test del sudor, sin embargo, ha mostrado ser más útil principalmente en tami- 
zaje, por lo que se ha planteado su reemplazo por el test de conductancia del sudor ${ }^{14}$. Otros métodos diagnósticos incluyen prueba de diferencia de potencial eléctrico nasal y medición de concentraciones de tripsinógeno en sangre entre otros ${ }^{15}$. Estudios más específicos involucran el diagnóstico molecular, especialmente en recién nacidos y niños pequeños con sospecha alta de fibrosis quística ${ }^{16}$. En este caso se realizo el diagnóstico por amplificación por PCR de ADN tomado de muestras de sangre de los gemelos. En cuanto al tratamiento de la enfermedad, en el pasado el abordaje se basaba en el manejo de las complicaciones emergentes de los diferentes sistemas comprometidos, con gran énfasis en las infecciones respiratorias, las cuales representan el $90 \%$ de la mortalidad. Sin embargo, en los últimos años se ha ampliado el campo de manejo teniendo en cuenta los mecanismos biológicos de la enfermedad, abriendo un camino a la búsqueda de una cura de la enfermedad, más allá de controlarla. Estas terapias en desarrollo incluyen terapia génica del CFTR, farmacoterapia dirigida al CFTR, terapias con agentes osmóticamente activos y anti-inflamatorios, y evolución del uso de antibióticos a forma inhalada ${ }^{17}$.

El compromiso del tracto gastrointestinal se presenta en el $85-90 \%$ de los casos, siendo la obstrucción intestinal postnatal temprana debido a íleo meconial una manifestación frecuente, presentándose como uno de los signos iníciales en el $10-15 \%$ de los pacientes $8,18,19$. Esta condición es el resultado de un espesamiento anormal del meconio causado por producción anormal de mucoproteinas (80-90\%), elevación en los niveles de calcio y la disfunción de los canales de cloro en la membrana del enterocito ${ }^{2,19}$. Este cuadro se ha asociado a otras complicaciones, incluyendo vólvulo intestinal y atresia intestinal hasta en un $50 \%$ de los pacientes ${ }^{8}$, situaciones que no se presentaron en este caso. Algunos autores asocian la presentación de íleo meconial con un fenotipo más severo y un peor pronóstico de la enfermedad ${ }^{5,20}$, sin embargo, en 2006 Pekcan et al. y Munck et al., publicaron estudios por separado, demostrando que estos afectados tienen el mismo riesgo de presentar infecciones pulmonares por el mismo tipo de bacterias, además de un mismo riesgo para el síndrome por obstrucción intestinal distal ${ }^{21,22}$.

Diferentes variaciones en el genotipo de la mutación del CFTR se han asociado a la manifestación fenotípica de la enfermedad, tal es el caso del compromiso pancreático ${ }^{6,13,23}$. Sin embargo, otras manifestaciones de la enfermedad no han logrado correlacionarse, indicando que hay factores por fuera del genotipo del CFTR que juegan un rol modulador en las complicaciones y curso de esta enfermedad de "un solo gen". Entre estos cuadros que no han sido correlacionados con la mutación del CFTR se incluyen los ya mencionados íleo meconial y síndrome por obstrucción intestinal distal, este último presentándose en niños un poco mayo$\operatorname{res}^{18,19,24,25}$. Estos factores reguladores podrían ser ambientales, como por ejemplo influenciados por alimentación o estadía en UCI, o gené$\operatorname{ticos}^{18,23}$; la presencia de diferencias fenotípicas en hermanos y hermanos gemelos hace pensar, sobretodo, en leves diferencias genotípicas. En el 2006, Blackman et al. Evaluaron estos posibles factores moduladores, encontrando que en el caso del íleo meconial existen regiones con genes sugestivamente modificadores, ya sea como predisponentes (cromosoma 4q35.1, $8 \mathrm{p} 23.1$ y $11 \mathrm{q} 25)$ o como protectores (20p11.22 y 21q22.3); mientras que en el caso del síndrome por obstrucción intestinal distal la presentación es modulada principalmente por factores ambientales ${ }^{18}$. Para el íleo meconial se han identificado polimorfismos de un solo nucleótido (SNP) en 3 genes de la familia de genes transportadores de solutos, SLC26A9 en el cromosoma 1, SLC9A3 en el cromosoma 5, y SLC6A14 en el cromosoma $\mathrm{X}^{10,26}$. Por otro lado, Bronsveld et al. Estudiaron la presencia de canales de cloro dependientes de cAMP codificados por CFTR en mellizos con fibrosis quística, encontrando que en algunos pacientes existe una función residual de estos canales, y que esta expresión tiende a ser más concordante entre gemelos monocigóticos que en aquellos dicigóticos ${ }^{27}$.

\section{Conclusiones}

La fibrosis quística ha sido reconocida como un desorden de un solo gen, sin embar- 
go, una diversidad considerable en su fenotipo y una amplia pleiotropía se ha evidenciado desde los primeros reportes de la enfermedad. En este caso se presentó una discordancia fenotípica importante en dos hermanos gemelos con el mismo genotipo para la enfermedad, hermanos que han estado expuestos a los mismos factores ambientales y comparten cerca al $100 \%$ de su ADN, sugiriendo que tan sólo pequeñas diferencias en su genoma puedan contribuir a la modulación de la expresión de la enfermedad. Cabe tener en cuenta la presencia e identificación de reguladores externos a esta mutación al momento de enfrentarse a este cuadro de expresividad variable, en especial en un momento en que empiezan a emerger diferentes tipos de terapia dirigidas a la búsqueda de cura de la enfermedad, más que el simple control de esta.

\section{Referencias}

1.- Diana A, Tesse R, Polizzi AM, et al: A large deletion causes apparent homozygosity for the $\mathrm{D} 1152 \mathrm{H}$ mutation in the cystic fibrosis transmembrane regulator (CFTR) gene. Gene 2012; 497 (1): 90-2.

2.- Rowe SM, Miller S, Sorscher EJ: Cystic fibrosis. N Engl J Med 2005; 352 (19): 1992-2001.

3.- Mall M, Kreda SM, Mengos A, et al: The Delta F508 mutation results in loss of CFTR function and mature protein in native human colon. Gastroenterology 2004; 126 (1): 32-41.

4.- Zielenski J, Rozmahel R, Bozon D, et al: Genomic DNA sequence of the cystic fibrosis transmembrane conductance regulator (CFTR) gene. Genomics 1991;10 (1): 214-28.

5.- Lai HJ, Cheng Y, Cho H, Kosorok MR, Farrell PM: Association between initial disease presentation, lung disease outcomes, and survival in patients with cystic fibrosis. Am J Epidemiol 2004; 159 (6): 537-46.

6.- Kristidis P, Bozon D, Corey M, et al: Genetic determination of exocrine pancreatic function in cystic fibrosis. Am J Hum Genet 1992; 50 (6): 1178-84.

7.- Kerem E, Corey M, Kerem BS, et al: The relation between genotype and phenotype in cystic fibrosis-analysis of the most common mutation (delta F508). N Engl J Med 1990; 323 (22): 1517-22.

8.- Agrons GA, Corse WR, Markowitz RI, Suárez ES, Perry $D R$ : Gastrointestinal manifestations of cystic fibrosis: radiologic-pathologic correlation. Radiographics. 1996; 16 (4): 871-93.

9.- Carels $K$, Claus F: Distal intestinal obstruction syndrome. JBR-BTR 2013; 96 (5): 318.

10.- Li W, Soave D, Miller MR, et al: Unraveling the complex genetic model for cystic fibrosis: pleiotropic effects of modifier genes on early cystic fibrosis-related morbidities. Hum Genet 2014; 133 (2): 151-61.

11.- Feuillet-Fieux MN, Lenoir G, Sermet I, et al: Nasal polyposis and cystic fibrosis $(\mathrm{CF})$ : review of the literature. Rhinology 2011; 49 (3): 347-55.

12.- Battezzati A, Mari A, Zazzeron L, et al: Identification of insulin secretory defects and insulin resistance during oral glucose tolerance test in a cohort of cystic fibrosis patients. Eur J Endocrinol 2011; 165 (1): 69-76.

13.- Borgo G, Mastella G, Gasparini P, Zorzanello A, Doro R, Pignatti PF: Pancreatic function and gene deletion F508 in cystic fibrosis. J Med Genet 1990; 27 (11): 665 9.

14.- Mattar AC, Leone C, Rodrigues JC, Adde FV: Sweat conductivity: An accurate diagnostic test for cystic fibrosis? J Cyst Fibros. 2014.

15.- Rueegg CS, Kuehni CE, Gallati S, et al: One-year evaluation of a neonatal screening program for cystic fibrosis in Switzerland. Dtsch Arztebl Int 2013; 110 (20): 356-63.

16.- van den Bergh FA, Martens A: [Diagnosis of cystic fibrosis; simple genotyping to rule out the disease preferable to starting with the sweat test]. Ned Tijdschr Geneeskd 2003; 147 (21): 1001-5.

17.- Amin R, Ratjen F: Emerging drugs for cystic fibrosis. Expert Opin Emerg Drugs 2014; 19 (1): 143-55.

18.- Blackman SM, Deering-Brose $R$, McWilliams $R$, et al: Relative contribution of genetic and nongenetic modifiers to intestinal obstruction in cystic fibrosis. Gastroenterology 2006; 131 (4): 1030-9.

19.- Gorter RR, Karimi A, Sleeboom C, Kneepkens CM, Heij $H A$ : Clinical and genetic characteristics of meconium ileus in newborns with and without cystic fibrosis. J Pediatr Gastroenterol Nutr 2010; 50 (5): 569-72.

20.- Evans AK, Fitzgerald DA, McKay KO: The impact of meconium ileus on the clinical course of children with cystic fibrosis. Eur Respir J 2001; 18 (5): 784-9.

21.- Pekcan S CN, Yalcin E, Dogru D, Ozcelik U, Kiper N: Comparing the clinical evolution of Cystic Fibrosis patients with meconium ileus and with non meconium ileus diagnosed under two months. Journal of Cystic Fibrosis 2006; 5 (1): S52.

22.- Munck A, Gérardin M, Alberti C, et al: Clinical outcome of cystic fibrosis presenting with or without meconium 
ileus: a matched cohort study. J Pediatr Surg 2006; 41 (9): 1556-60.

23.- Salvatore F, Scudiero O, Castaldo G: Genotype-phenotype correlation in cystic fibrosis: the role of modifier genes. Am J Med Genet 2002; 111 (1): 88-95.

24.- Subhi R, Ooi R, Finlayson F, et al: Distal intestinal obstruction syndrome in cystic fibrosis: presentation, outcome and management in a tertiary hospital (20072012). ANZ J Surg 2013.

25.- De Lisle RC, Borowitz D: The cystic fibrosis intestine.
Cold Spring Harb Perspect Med 2013; 3 (9): a009753.

26.- Sun L, Rommens JM, Corvol H, et al: Multiple apical plasma membrane constituents are associated with susceptibility to meconium ileus in individuals with cystic fibrosis. Nat Genet 2012; 44 (5): 562-9.

27.- Bronsveld I, Mekus F, Bijman J, et al: Residual chloride secretion in intestinal tissue of delta F508 homozygous twins and siblings with cystic fibrosis. The European CF Twin and Sibling Study Consortium. Gastroenterology 2000; 119 (1): 32-40. 